\title{
Differential expression of CD34 in normal colorectal tissue, peritumoral inflammatory tissue, and tumour stroma
}

\author{
Hirofumi Nakayama, Hideaki Enzan, Eriko Miyazaki, Naoto Kuroda, Keishi Naruse, \\ Makoto Hiroi
}

\begin{abstract}
Aims-To investigate the role of CD34 positive stromal cells, namely dendritic interstitial cells, in the desmoplastic stroma formation of malignant epithelial neoplasms the distribution of $\mathrm{CD} 34$ positive stromal cells was examined in human colorectal adenocarcinomas, peritumoral inflammatory tissue, and normal tissue.

Methods-Forty one surgically resected human colorectal adenocarcinomas and their corresponding peritumoral inflammatory and normal tissues were examined. To distinguish CD34 positive stromal cells from vascular endothelial cells, immunostaining for both CD34 and CD31 was performed. The distribution of myofibroblasts was also analysed immunohistochemically, and double staining with CD34 and $\alpha$ smooth muscle actin (ASMA) was performed.
\end{abstract}

Results-Most of the stromal cells in the normal colorectal submucosa, muscularis propria, subserosa, and perirectal tissue were positive for CD34. In contrast, the peritumoral inflammatory tissue and the tumour stroma had no CD34 positive stromal cells. The distribution of myofibroblasts was almost the same as in the aforementioned series. No stromal cells double positive for CD34 and ASMA were detected in the peritumoral inflammatory tissues.

Conclusions-Most stromal fibroblasts are CD34 positive stromal cells (dendritic interstitial cells). In colorectal adenocarcinomas, a lack of CD34 expression in stromal cells is associated with desmoplastic reaction.

(f Clin Pathol 2000;53:626-629)

Keywords: CD34; dendritic interstitial cell; colon carcinoma; rectal carcinoma

Human progenitor cell antigen (CD34) positive stromal cells, which are termed dendritic interstitial cells, ${ }^{1}$ are distributed throughout the human body including the breast, ${ }^{2}$ thyroid gland, ${ }^{3}$ submandibular gland, ${ }^{4}$ dermis, ${ }^{5}$ uterine stroma,${ }^{6}$ and intestine. ${ }^{7}$ CD 34 positive stromal cells (dendritic interstitial cells) are thought to play a supportive role not only in the maturation and proliferation of adjacent mesenchymal and epithelial stem cells, ${ }^{1}$ but also in immune mediated responses. ${ }^{1-4} \mathrm{CD} 34$ positive stromal cells are distinct from the interstitial cells of Cajal. ${ }^{7}$
Several studies have been performed to investigate the distribution of $\alpha$ smooth muscle actin (ASMA) positive stromal cells (myofibroblasts) in colorectal and breast epithelial neoplasms..$^{8-12}$ A recent study has revealed that myofibroblasts in the liver are derived from Ito cells/hepatic stellate cells. ${ }^{13}{ }^{14}$ The myofibroblasts in the liver are detected in hepatocellular carcinomas, ${ }^{13}{ }^{14}$ cholangiocellular carcinomas, and metastatic liver carcinomas. ${ }^{15}$ Moreover, it is indicated that myofibroblasts are associated with capsular collagen formation in hepatocellular carcinomas. ${ }^{16}$ Apart from in the liver, however, the origin of myofibroblasts is still controversial.

The relation between CD34 positive stromal cells and desmoplastic stroma of malignant epithelial neoplasms has not been studied extensively. Recently, we have reported that CD34 positive stromal cells (dendritic interstitial cells) and myofibroblasts show a bilayered structure at the periphery of major salivary gland pleomorphic adenomas. ${ }^{17}$

In our present study, we examined the distribution of CD34 positive stromal cells (dendritic interstitial cells) in colorectal adenocarcinomas, peritumoral inflammatory tissues, and normal tissues to elucidate the importance of CD34 positive stromal cells in desmoplastic stroma formation.

\section{Materials and methods}

We selected 41 surgically resected invasive colorectal adenocarcinomas and their corresponding peritumoral inflammatory and normal tissues from the surgical pathology files of the first department of pathology, Kochi Medical School and its affiliated hospitals from 1997 to 1998 (32 tumours invading the subserosa or perirectal tissue, five confined to the muscularis propria, and four confined to the submucosa). All of the specimens selected were fixed with $10 \%$ buffered formalin immediately after resection. All of the 41 colorectal adenocarcinomas were well or moderately differentiated.

Immunohistochemical studies were performed by the streptavidin-biotin method using the Histofine SAB-PO $(M)$ kit (Nichirei, Tokyo, Japan). Four monoclonal antibodies against CD34, CD31, ASMA, and high molecular weight caldesmon (HCD) were used. Table 1 lists the monoclonal antibodies and staining procedures used. We examined CD31 immunoreactivity in all of the tumours and their peritumoral inflammatory and normal tissues, to distinguish CD34 positive 
Table 1 Antibodies used for immunohistochemical analysis

\begin{tabular}{llll}
\hline $\begin{array}{l}\text { Antibody } \\
\text { (clone) }\end{array}$ & Specificity & Sources & $\begin{array}{l}\text { Working dilution } \\
\text { (antigen retrieval) }\end{array}$ \\
\hline MY10 & CD34 & Becton-Dickinson, San Jose, California, USA & $1 / 20$ (none) \\
JC/70A & CD31 & DAKOpatts, Glostrup, Denmark & $1 / 20$ (pronase) \\
1A4 & $\alpha$ Smooth muscle actin (ASMA) & DAKOpatts & $1 / 50$ (none) \\
h-CD & High molecular weight caldesmon (HCD) & DAKOpatts & $1 / 50$ (microwave) \\
\hline
\end{tabular}

These four antibodies are mouse monoclonal antibodies directed against human tissue.

stromal cells (dendritic interstitial cells) from vascular endothelial cells, which are positive for both CD34 and CD $31 .{ }^{18}$ Vascular endothelial cells were used as the internal positive control of immunostaining for CD34 and CD31. We classified ASMA positive and HCD negative stromal cells as myofibroblasts. ${ }^{12}$

After immunostaining, we examined the distribution of CD34 positive stromal cells (dendritic interstitial cells) in colorectal adenocarcinomas, peritumoral inflammatory tissues, and normal tissues of the 41 cases. In general, peritumoral tissues were inflamed and the degree of inflammation varied from one tumour to another. We classified areas adjacent to the tumour and containing myofibroblasts and band like inflammatory infiltrates as peritumoral inflammatory tissues; we also classified areas that were located in the outer side of the peritumoral inflammatory tissues as normal colorectal tissues.

We also graded the number of myofibroblasts within the tumours, the peritumoral inflammatory tissues, and the normal tissues, as we had described in one of our previous reports. ${ }^{12}$

Double immunostaining for CD34 and ASMA was performed; CD34 and ASMA were labelled with 3,3'-diaminobenzidine tetrachloride (DAB) and alkaline phosphatase fast blue, respectively.

\section{Results}

Table 2 shows the results of the immunohistochemical analysis of the distribution of CD34 positive stromal cells in the 41 colorectal adenocarcinomas, peritumoral inflammatory tissues, and normal colorectal tissues.

CD34 positive stromal cells (dendritic interstitial cells) had small round to oval nuclei and little cytoplasm; they were positive for CD34 but negative for CD31.

In all of the 41 cases examined, most of the stromal cells were positive for CD34 and negative for CD31 in the normal submucosa, muscularis propria, subserosa, and perirectal tis-

Table 2 The distribution of CD34 positive stromal cells (dendritic interstitial cells) in the tumour stroma, peritumoral inflammatory tissues, and normal tissues of the 41 invasive colorectal adenocarcinoma cases

\begin{tabular}{llllr}
\hline & & \multicolumn{2}{l}{$\begin{array}{l}\text { CD34 positive stromal cells } \\
\text { (dendritic interstitial cells) }\end{array}$} \\
\cline { 3 - 5 } $\begin{array}{l}\text { Tumour } \\
\text { depth }\end{array}$ & $\begin{array}{l}\text { Number } \\
\text { of cases }\end{array}$ & $T$ & $B$ & \multicolumn{1}{l}{$N$} \\
\hline Sm & 4 & 0 & 0 & $4(100 \%)$ \\
Mp & 5 & 0 & 0 & $5(100 \%)$ \\
Ss & 32 & 0 & 0 & $32(100 \%)$ \\
Total & 41 & 0 & 0 & $41(100 \%)$ \\
\hline
\end{tabular}

$\mathrm{Sm}$, submucosa; $\mathrm{Mp}$, muscularis propria; $\mathrm{Ss}$, subserosa or perirectal tissue; $\mathrm{T}$, tumour stroma; $\mathrm{B}$, peritumoral inflammatory tissue; $\mathrm{N}$, normal colorectal tissue. sue; no CD34 positive stromal cells were detected in the mucosa. CD34 positive stromal cells were mainly in the perivascular area of the normal submucosa and subserosa (figs 1 and 2; note: figs 1 and 2 are from the same site). CD34 positive stromal cells (dendritic interstitial cells) were also detected adjacent to the smooth muscle bundles forming the muscularis propria and muscularis mucosa.

In contrast, all of the colorectal adenocarcinomas examined had no CD34 positive stromal cells; CD34 were positive only in vascular endothelial cells (fig 3 ).

The peritumoral inflammatory tissues showed non-specific inflammatory change, the degree of which varied from one case to another, and from one area to another in each case. In all of the cases examined, the peritumoral tissues had no CD34 positive stromal cells (fig 4).

With regard to myofibroblasts, the results were almost the same as previous reports, including our own. ${ }^{912} 19$ All of the 41 tumours had a large number of myofibroblasts within the tumours, forming desmoplastic stroma. Three of the four cases with tumours confined to the submucosa had a moderate or large number of myofibroblasts in the peritumoral

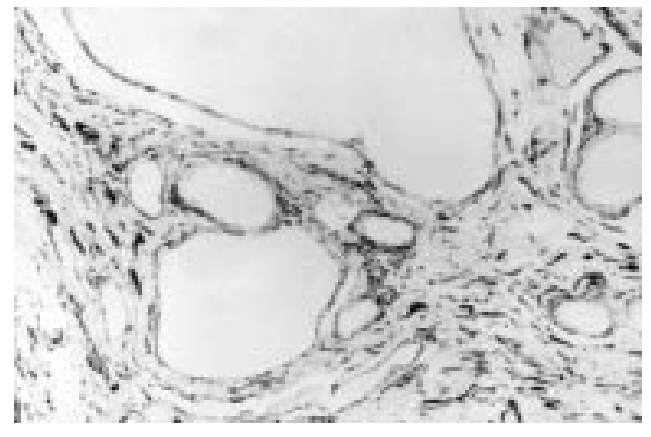

Figure 1 CD34 immunostaining in normal colorectal tissue. CD34 positive stromal cells (dendritic interstitial cells) are detected.

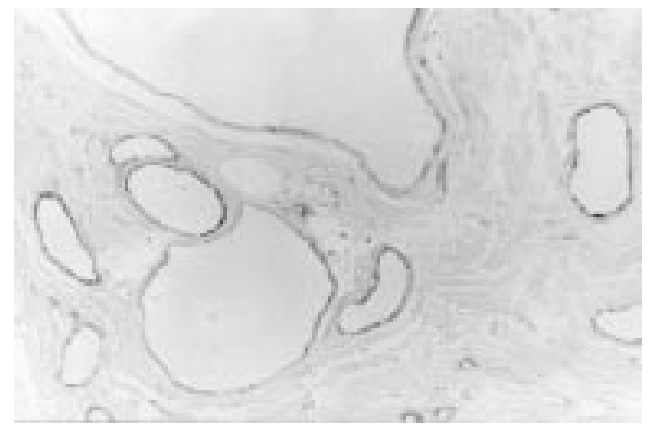

Figure 2 CD31 immunostaining in normal colorectal tissue. CD31 expression is restricted to vascular endothelial cells; CD34 positive stromal cells (dendritic interstitial cells) are negative for CD31. 


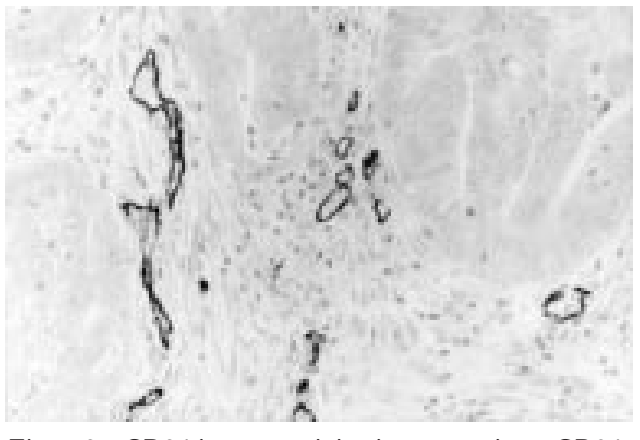

Figure 3 CD34 immunostaining in tumour tissue. CD34 is positive in vascular endothelial cells; no CD34 positive stromal cells are seen.

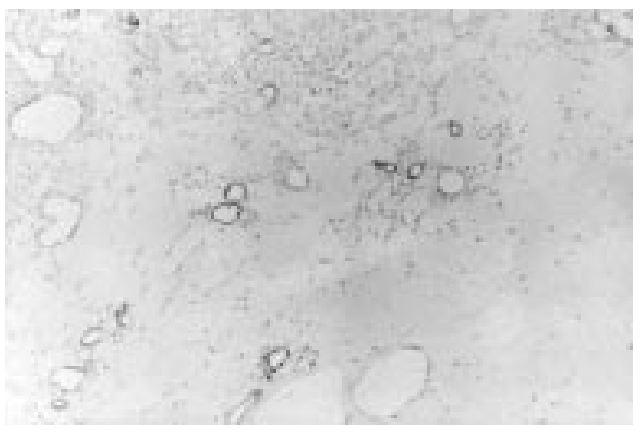

Figure 4 CD34 immunostaining in the peritumoral tissue showing non-specific inflammatory change. CD34 is positive in vascular endothelial cells; no CD34 positive stromal cells are seen.

inflammatory tissue; the remaining one had a small number of myofibroblasts in the peritumoral inflammatory tissue. All of the five cases with tumours confined to the muscularis propria had a small number of myofibroblasts in the peritumoral inflammatory tissue. Twenty seven of the 32 cases with tumours invading the subserosa/perirectal tissue had a moderate or large number of myofibroblasts in the peritumoral inflammatory tissue in the subserosa; all of the 32 cases had a small number of myofibroblasts in the peritumoral inflammatory tissue in the submucosa and the muscularis propria. Except for pericryptal fibroblasts, there were no ASMA positive stromal cells in the normal colorectal tissue examined.

No stromal cells double positive for CD34 and ASMA were detected at the peritumoral inflammatory tissues examined.

\section{Discussion}

It has been reported previously that the murine fibroblast cell line $3 \mathrm{~T} 3$ shows strong immunoreactivity for CD $34 .^{20}$ In the field of dermatopathology, there appears to be an inverse correlation between CD34 expression and proline-4-hydroxylase immunoreactivity by stromal cells; dermal stromal cells in hypertrophic scars and those at the inflammatory expanding borders of keloids lose CD34 expression, but they strongly express proline-4-hydroxylase. ${ }^{21}$ The increase in proline-4-hydroxylase expression correlates with the active synthesis of type I collagen, ${ }^{21}$ namely myofibroblastic transformation. Therefore, CD34 positive stromal cells (dendritic interstitial cells) show a transition to myofi- broblasts in the skin. ${ }^{21}$ Wound healing and the generation of tumour stroma share many important properties. ${ }^{22}$ The recognised differences between tumour stroma generation and wound healing are minor. ${ }^{22}$ Desmoplasia in malignant epithelial tumours resembles wound healing, which results in hypertrophic scars and keloids. ${ }^{22}$

Regarding the origin of myofibroblasts in the human colorectum, it was suggested that myofibroblasts in malignant epithelial tumour stroma are derived from stromal fibroblasts, ${ }^{23}$ smooth muscle cells, ${ }^{23}$ and pericryptal fibroblasts ${ }^{11}$; the most likely situation is that myofibroblasts arise from several cells including fibroblasts, vascular smooth muscle cells, pericytes, and other specialised mesenchymal cells with myoid features, among which fibroblasts predominate. ${ }^{24}$ Recently, it has been reported that CD34 positive stromal cells in the human intestine express proline4-hydroxylase, ${ }^{7}$ suggesting that stromal fibroblasts are identical to CD34 positive stromal cells. In our study, most of the stromal cells in the normal colorectal submucosa, muscularis propria, and subserosa were positive for CD34; the desmoplastic tumour stroma had no CD34 positive stromal cells. In several articles, including our own, stromal cells in malignant colorectal epithelial tumours were found to be ASMA positive, indicating that they are myofibroblasts. ${ }^{19}$ In addition, we have reported previously that myofibroblasts surround colorectal adenocarcinomas. ${ }^{12}$ In general, except for pericryptal fibroblasts, there are no ASMA positive stromal cells in normal colorectal tissues. ${ }^{19}$ No stromal cells double positive for CD34 and ASMA were detected, suggesting that a lack of CD34 expression is essential for the gain of ASMA expression in desmoplastic stroma of colorectal adenocarcinomas.

Colorectal adenomas have no desmoplastic stroma. The total absence of CD34 positive stromal cells (dendritic interstitial cells) in the mucosa of normal colorectal tissues is associated with lack of desmoplasia in colorectal adenomas.

In conclusion, desmoplastic stroma and peritumoral inflammatory tissues had no CD34 positive stromal cells. In colorectal adenocarcinomas, a lack of CD34 expression in stromal cells is associated with the desmoplastic reaction. To elucidate the association between CD34 positive stromal cells (dendritic interstitial cells) and myofibroblasts further comprehensive studies are needed.

The authors are grateful to Ms Miko Mitani, Ms Hisayo Yamasaki, Ms Nao Nakamura, and Mr Tadatoshi Tokaji, first department of pathology, Kochi Medical School; Mr Masatoshi Shirota, medical research center, Kochi Medical School; and Ms Yoshi Nabeshima, department of pathology, Chikamori Hospital for their excellent technical assistance. This work was supported by the Foundation for Promotion of Cancer Research in Japan.

1 von de Rijn M, Rouse RV. CD34. A review. Applied Immunohistochemistry 1994;2:71-80.

2 Yamazaki K, Eyden BP. Ultrastructural and immunohistochemical observations on intralobular fibroblasts of human breast, with observations on the CD34 antigen. f breast, with observations on the CD

3 Yamazaki K, Eyden BP. Ultrastructural and immunohistochemical studies of intralobular fibroblasts in human submandibular gland: the recognition of a CD34-positive 
stromal cell network communicated by gap junctions. $\mathcal{F}$ Submicrosc Cytol Pathol 1996;28:471-83.

4 Yamazaki K, Eyden BP. Ultrastructural and immunohistochemical studies of intralobular fibroblasts in human thyroid gland: recognition of a CD34-positive stromal cel network communicated by gap junctions and terminated by autonomic nerve endings. F Submicrosc Cytol Pathol 1997;29:461-76.

5 Nickoloff BJ. The human progenitor cell antigen (CD34) is localized on endothelial cells, dermal dendritic cells, and perifollicular cells in formalin-fixed normal skin, and on proliferating endothelial cells and stromal spindle-shaped cells in Kaposi's sarcoma. Arch Dermatol 1991;127:523-9.

6 Lindenmayer AE, Miettinen M. Immunophenotypic features of uterine stromal cells: CD34 expression in endocervical stroma. Virchows Arch 1995;426:457-60.

7 Vanderwinden JM, Rumessen JJ, De Laet MH, et al. CD34+ cells in human intestine are fibroblasts adjacent to, but distinct from, interstitial cells of Cajal. Lab Invest 1999;79:59tinct

8 Lazard D, Sastre X, Frid MG, et al. Expression of smooth muscle-specific proteins in myoepithelium and stromal myofibroblasts of normal and malignant human breast tis999-1003.

9 Hewitt RE, Powe DG, Carter GI, et al. Desmoplasia and its relevance to colorectal tumor invasion. Int $\mathcal{F}$ Cancer 1993;53:62-9.

Lagace R, Schurch W, et al. Myofibroblasts in the stroma of invasive and metastatic carcinoma. A possible host response to neoplasia. Am F Surg Pathol 1979;3:52533.

11 Sappino AP, Dietrich PY, Skalli O, et al. Colonic pericryptal fibroblasts. Differentiation pattern in embryogenesis and phenotypic modulation in epithelial proliferative lesions.
Virchows Arch A Pathol Anat Histopathol 1989;415:551-7.

12 Nakayama H, Enzan H, Miyazaki E, et al. The role of myofibroblasts at the tumor border of invasive colorectal adenocarcinomas. $\not$ pn $\mathcal{F}$ Clin Oncol 1998;28:615-20.

13 Enzan H, Himeno H, Iwamura S, et al. Immunohistochemical identification of Ito cells and their myofibroblaschemical identification of Ito cells and their myofibroblastic transformation
14 Enzan H, Himeno H, Iwamura S, et al. Alpha-smooth muscle actin-positive perisinusoidal cells in human hepatocelcle actin-positive perisinusoidal cells in human
lular carcinomas. Hepatology 1994;19:895-903.

15 Terada T, Mikimoto K, Terayama N, et al. Alpha-smooth muscle actin-positive stromal cells in cholangiocellular carcinomas, hepatocellular carcinomas and metastatic liver carcinomas. f Hepatol 1996;24:706-12.

16 Ooi LPJ, Crawford DHG, Gotley DC, et al. Evidence that "myofibroblast-like" cells are the cellular source of capsular collagen in hepatocellular carcinoma. $\mathcal{F}$ Hepatol 1997;26: 798-807.

17 Nakayama $\mathrm{H}$, Naruse $\mathrm{K}$, Miyazaki $\mathrm{E}$, et al The specific distribution of dendritic interstitial cells at the tumor border of major salivary gland pleomorphic adenomas. Mod Pathol 1999;12:445-9.

18 Miettinen M, Lindenmayer AE, Chaubal A. Endothelial cell markers CD 31, CD34, and BNH9 antibody to $\mathrm{H}-$ and Y-antigens: evaluation of their specificity and sensitivity in the diagnosis of vascular tumours and comparison with von Willebrand factor. Mod Pathol 1994;7:82-90.

19 Nakayama H, Miyazaki E, Enzan H. Differential expression of high-molecular-weight caldesmon in colorectal pericryptal fibroblasts and tumour stroma. F Clin Pathol 1999;52. $785-6$.

20 Lin G, Finger E, Gutierrez RJ. Expression of CD34 in endothelial cells, hematopoietic progenitors and nervous cells in fetal and adult mouse tissues. Eur 7 Immunol 1995; 25:1508-16.

21 Aiba S, Tagami H. Inverse correlation between CD34 expression and proline-4-hydroxylase immunoreactivity on pindle cells noted in hypertrophic scars and keloids. $\mathcal{F}$ Cutan Pathol 1997;24:65-9.

22 Dvorak HF. Tumor: wounds that do not heal. Similarities between tumor stroma generation and wound healing. $N$ Engl f Med 1986;315:1650-9.

23 Ohtani H, Sasano N. Stromal cell changes in human colorectal adenomas and carcinomas. Virchows Arch A Pathol Anat Histopathol 1983;401:209-22.

24 Schurch W, Seemayer TA, Gabbiani G. Myofibroblasts. In: Sternberg SS, ed. Histology for pathologists, 2nd ed. New York: Lippincott-Raven Publishers, 1997:127-65. 